\title{
A nomogram for predicting overall survival in patients with resected non-small cell lung cancer treated with chemotherapy
}

\author{
Yuan Zeng ${ }^{1,2 \#}$, Nicholas Mayne ${ }^{3 \#}$, Chi-Fu Jeffrey Yang ${ }^{3 \#}$, Jun Liu ${ }^{1,2}$, Fei Cui ${ }^{1,2}$, Jingpei Li ${ }^{1,2}$, \\ Wenhua Liang $^{1,2}$, Jianxing $\mathrm{He}^{1,2}$; written on behalf of the AME Thoracic Surgery Collaborative Group \\ ${ }^{1}$ Department of Thoracic Surgery, the First Affiliated Hospital of Guangzhou Medical University, Guangzhou, China; ${ }^{2}$ Guangzhou Institute \\ of Respiratory Disease \& China State Key Laboratory of Respiratory Disease \& National Clinical Research Center for Respiratory Disease, \\ Guangzhou, China; ${ }^{3}$ Section of General Thoracic Surgery, Duke University Medical Center, Durham, NC, USA \\ Contributions: (I) Conception and design: J He, Y Zeng, W Liang; (II) Administrative support: J He, N Mayne; (III) Provision of study materials or \\ patients: J He, Y Zeng, W Liang, N Mayne, CFJ Yang; (IV) Collection and assembly of data: J He, Y Zeng, W Liang, N Mayne, CFJ Yang; (V) Data \\ analysis and interpretation: All authors; (VI) Manuscript writing: All authors; (VII) Final approval of manuscript: All authors. \\ "These authors contributed equally to this work. \\ Correspondence to: Wenhua Liang, MD; Jianxing He, MD, PhD, FACS. Department of Thoracic Surgery, the First Affiliated Hospital of Guangzhou \\ Medical University; Guangzhou Institute of Respiratory Disease \& China State Key Laboratory of Respiratory Disease, No. 151, Yanjiang Rd, \\ Guangzhou 510120, China. Email: liangwh1987@163.com; drhe_jianxing@163.com.
}

Background: Chemotherapy is a common treatment for patients with resected non-small cell lung cancer (NSCLC). However, there are few models for predicting the survival outcomes of these patients. Here, we developed a clinical nomogram for predicting overall survival (OS) in this cohort.

Methods: A total of 16,661 patients with resected NSCLC treated with chemotherapy were extracted from the Surveillance, Epidemiology, and End Results (SEER) database. We identified prognostic factors and integrated them into a nomogram. The model was subjected to bootstrap internal validation using the SEER database and external validation using a database in China and the National Cancer Database (NCDB). The model's predictive accuracy and discriminative ability were tested by calibration and concordance index (C-index).

Results: Age, sex, number of dissected lymph nodes, extent of surgery, $\mathrm{N}$ stage, T stage, and grade were independent factors for OS and were integrated into the model. The calibration curves for probability of 1-, 3-, and 5-year OS showed excellent agreement between the predicted and actual survivals. The C-index of the nomogram was higher than that of the Tumor-Node-Metastasis staging system for predicting OS (training cohort, 0.62 vs. 0.58; China cohort, 0.68 vs. 0.63 ; NCDB cohort, 0.59 vs. 0.57).

Conclusions: We developed a nomogram that can present individual prediction of OS for patients with resected NSCLC who are undergoing chemotherapy. This practical prognostic tool may help clinicians in treatment planning.

Keywords: Non-small cell lung cancer (NSCLC); nomogram; chemotherapy

Submitted Dec 01, 2020. Accepted for publication Feb 07, 2021.

doi: $10.21037 /$ tlcr-20-1220

View this article at: http://dx.doi.org/10.21037/tlcr-20-1220

\section{Introduction}

Lung cancer is the most commonly diagnosed cancer (11.6\% of total cases) and the leading cause of cancerrelated death (18.4\% of total cancer deaths) worldwide (1). For NSCLC patients without distant metastases, including patients with stage I and II disease and a subset of patients with stage III disease, the standard curative treatment is radical resection (2). In the $8^{\text {th }}$ edition of the Tumor-NodeMetastasis (TNM) classification, adjuvant chemotherapy is also recommended for stage II-III patients according to 
the National Comprehensive Cancer Network (NCCN) guidelines (2). In addition, adjuvant chemotherapy is occasionally administered in high-risk patients with stage I NSCLC $(3,4)$. Further, some stage III patients undergo chemotherapy before surgery (5). Consequently, there is a large proportion of patients with resected NSCLC undergoing chemotherapy in the real world.

The TNM classification is stratified based on tumor size and lymph node involvement in resected NSCLC patients. However, there are various other independent prognostic factors, such as sex, age, tumor histology, tumor differentiation, and number of dissected lymph nodes, that may contribute considerably to individualized predictions of survival $(4,6)$. A nomogram allows for such individualized survival prediction $(6,7)$. Established nomograms for NSCLC have provided a more accurate prediction than traditional TNM staging systems $(6,8,9)$. However, there are few models for predicting the survival of patients with resected NSCLC receiving chemotherapy. In this study, our aim was to develop a clinical nomogram for predicting overall survival (OS) in patients with resected NSCLC undergoing chemotherapy based on the data from the Surveillance, Epidemiology, and End Results (SEER) database. Furthermore, we used external validation cohorts from China and the National Cancer Database (NCDB) to validate the nomogram. We present the following article in accordance with the TRIPOD reporting checklist (available at http://dx.doi.org/10.21037/tlcr-20-1220).

\section{Methods}

\section{Patient selection criteria}

Details of patients with resected NSCLC undergoing chemotherapy between 2004 and 2015 were extracted from the SEER-18 database using SEER`Stat software (v8.3.5). The inclusion criteria were as follows: pathologically confirmed primary NSCLC between January 2004 and December 2015; history of lobectomy, pneumonectomy, segmentectomy, or wedge resection as curative treatment for lung cancer; treatment with chemotherapy; and the presence of one malignant primary lesion. The exclusion criteria were as follows: distant metastasis; invasion of the diaphragm, mediastinum, heart, great vessels, trachea, recurrent laryngeal nerve, esophagus, vertebral body, or carina; N3 disease; those who died within 30 days of surgery; and incomplete information. A standardized data form was created to obtain all relevant data, including age, sex, tumor size, $T$ stage, tumor location, examined lymph node, $\mathrm{N}$ stage, differentiation, histological type, extent of surgery, radiation and VPI (visceral pleural invasion). The TNM staging system was characterized according to the sixth edition of the American Joint Committee on Cancer (AJCC). Histology and tumor location codes were listed in Table S1.

To assess the generalizability of the model, two external validation cohorts that met the inclusion and exclusion criteria were used to examine the applicability of the model. One cohort was composed of 1,108 patients from the First Affiliated Hospital of Guangzhou Medical University, China between 2009 and 2014. The other cohort comprised 43,767 patients from the NCDB between 2000 and 2015 . The NCDB is a joint project of the Commission on Cancer (CoC) of the American College of Surgeons and the American Cancer Society. The CoC's NCDB and the hospitals participating in the $\mathrm{CoC} \mathrm{NCDB}$ are the sources of the de-identified data used herein; they have not verified and are not responsible for the statistical validity of the data analysis or the conclusions derived by the authors. The data is considered as hospital-based rather than populationbased. All the data and analysis using NCDB were conducted by Nicholas Mayne and Chi-Fu Jeffrey Yang in Duke University Medical Center. All procedures performed in this study were in accordance with the Declaration of Helsinki (as revised in 2013).

\section{Construction of the nomogram}

The training dataset $(n=16,661)$ from SEER was used for the original nomogram development. OS was calculated using the Kaplan-Meier method and compared using the log-rank test. Multivariable Cox proportional hazard regression was used to identify independent prognostic factors. Based on the significant independent factors, nomograms were developed using R 3.5.3 (http://www. r-project.org) with the survival and rms packages (10).

\section{Validation and calibration of the nomogram}

The model was subjected to bootstrap internal validation in the training cohort and external validation with the cohorts from the First Affiliated Hospital of Guangzhou Medical University and the NCDB. The discrimination ability was identified by applying the concordance index (C-index) (11). Calibration for the 1-, 3-, and 5-year OS, which compared the predicted survival with the observed survival, was 
performed with a calibration curve.

\section{Statistical analysis}

The chi-square test was used to evaluate the statistical significance of differences in the proportions of variables for the three cohorts. Overall survival (OS) was defined as the time from medical diagnosis to death from any cause. OS were estimated using the Kaplan-Meier method and compared using the log-rank test. Multivariate Cox proportional hazard regression was used to determine independent prognostic factors. The hazard ratio (HR) and corresponding $95 \%$ confidence interval (CI) were calculated. Statistical analyses were performed using SPSS 22.0. The C-indexes of two different models were compared as previously described (12). Statistical analyses were performed utilizing MedCalc 18.2.1. $\mathrm{P}<0.05$ was considered statistically significant.

\section{Results}

\section{Clinical characteristics}

A total of 16,661 patients with resected NSCLC treated with chemotherapy from the SEER database met the inclusion criteria. The median (interquartile range) follow-up was 27 months (13-52 months). For the external validation cohorts, there were 1,108 patients from hospitals in China and 43,767 patients from the NCDB. The median followup times in these cohorts were 41 months (28-59 months) and 36 months (22-49 months), respectively. Table 1

Table 1 Demographics and clinicopathologic characteristics of the training and external validation cohorts

\begin{tabular}{|c|c|c|c|c|c|c|c|}
\hline \multirow[t]{2}{*}{ Characteristic } & \multicolumn{2}{|c|}{ Training cohort $(n=16,661)$} & \multicolumn{2}{|c|}{$\begin{array}{l}\text { External validation cohort (China) } \\
\qquad(\mathrm{n}=1108)\end{array}$} & \multicolumn{2}{|c|}{$\begin{array}{l}\text { External validation cohort } \\
\qquad(\mathrm{NCDB})(\mathrm{n}=43767)\end{array}$} & \multirow[t]{2}{*}{$\mathrm{P}$} \\
\hline & No. & $\%$ & No. & $\%$ & No. & $\%$ & \\
\hline Age & & & & & & & $<0.001$ \\
\hline$<60$ & 5,488 & 32.9 & 512 & 46.2 & 12,652 & 28.9 & \\
\hline $60-70$ & 6,601 & 39.6 & 394 & 35.6 & 16,985 & 38.8 & \\
\hline$\geq 70$ & 4,572 & 27.4 & 202 & 18.2 & 14,130 & 32.3 & \\
\hline Gender & & & & & & & $<0.001$ \\
\hline Male & 8,711 & 52.3 & 656 & 59.2 & 21,733 & 49.7 & \\
\hline Female & 7,950 & 47.7 & 452 & 40.8 & 22,034 & 50.3 & \\
\hline \multicolumn{8}{|l|}{ Race } \\
\hline White & 13,801 & 82.8 & - & - & - & - & \\
\hline Black & 1,610 & 9.7 & - & - & - & - & \\
\hline Other & 1,250 & 7.5 & - & - & - & - & \\
\hline Location & & & & & & & $<0.001$ \\
\hline Upper & 9,951 & 59.7 & 558 & 50.4 & 25,118 & 57.4 & \\
\hline Middle & 771 & 4.6 & 69 & 6.2 & 1,996 & 4.6 & \\
\hline Lower & 5217 & 31.3 & 415 & 37.5 & 14,285 & 32.6 & \\
\hline Other & 722 & 4.3 & 66 & 5.9 & 2,368 & 5.4 & \\
\hline Examined lymph node & & & & & & & $<0.001$ \\
\hline $0-9$ & 8,361 & 50.2 & 286 & 25.8 & 21,425 & 49 & \\
\hline $10-15$ & 4,260 & 25.6 & 238 & 21.5 & 11,627 & 26.6 & \\
\hline$\geq 16$ & 4,040 & 24.2 & 584 & 52.7 & 10,715 & 24.5 & \\
\hline
\end{tabular}

Table 1 (continued) 
Table 1 (continued)

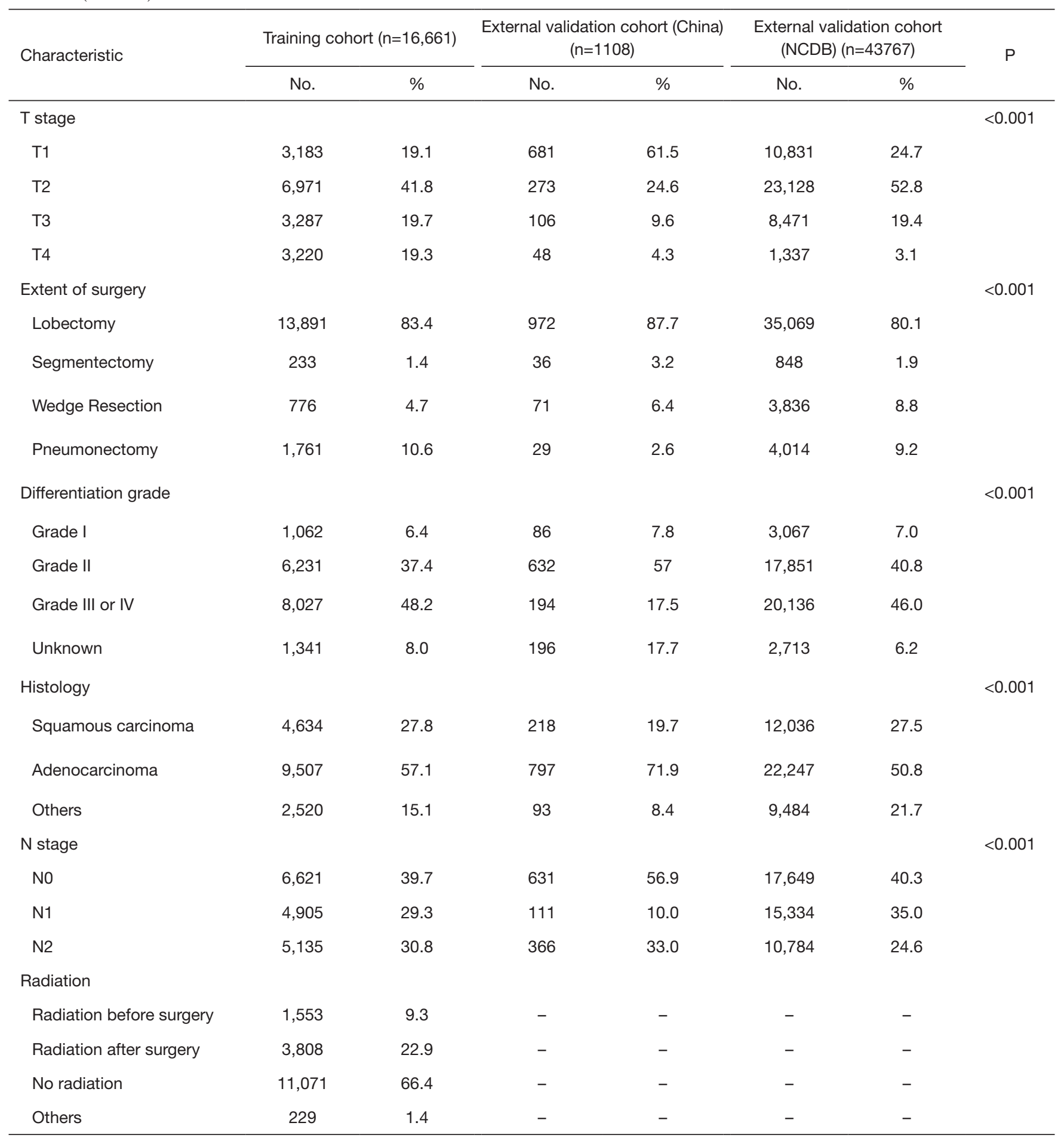


summarizes the demographics and clinicopathological characteristics in the three cohorts.

\section{Independent prognostic factors in the training cohort}

The outcomes of the univariate analysis are shown in Table 2. All significant predictors of overall survival in the univariate analysis were accessed into the multivariate analysis. The multivariate analysis showed that age $(\mathrm{P}<0.001)$, sex $(\mathrm{P}<0.001)$, primary site $(\mathrm{P}<0.001)$, number of dissected lymph nodes $(\mathrm{P}<0.001)$, extent of surgery $(\mathrm{P}<0.001)$, differentiation $(\mathrm{P}<0.001)$, histology $(\mathrm{P}=0.031), \mathrm{T}$ stage $(\mathrm{P}<0.001)$, and $\mathrm{N}$ stage $(\mathrm{P}<0.001)$ were independent prognostic factors for OS (Table 2).

\section{Development of a prognostic nomogram}

Significant independent factors, including age, sex, primary site, number of dissected lymph nodes, extent of surgery, differentiation, histology, $\mathrm{T}$ stage, and $\mathrm{N}$ stage, were integrated to create the nomogram (Figure 1). The nomogram demonstrated $\mathrm{N}$ stage, $\mathrm{T}$ stage, and age as having the greatest contribution to prognosis, followed by extent of surgery, gender, and differentiation. The number of dissected lymph nodes, primary site, and histology showed a relatively small impact on survival. By calculating the total score and comparing it to the highest possible score, it was possible to obtain the predicted 1-, 3-, and 5 -year OS.

\section{Calibration and validation of the nomogram}

The calibration plots indicated excellent agreement between the nomogram prediction and the actual observation for 1-, 3-, and 5-year OS in the training cohort (Figure 2). The calibration plots also showed acceptable agreement in the external validation cohorts. In the training cohort, the Harrell's C-index for the nomogram to predict OS [0.62; $95 \%$ confidence interval (CI), 0.61-0.63] was considerably greater than that of the American Joint Committee on Cancer $8^{\text {th }}$ edition TNM staging system (stage IA, IB, IIA, IIB, IIIA, and IIIB) $(0.58$; 95\% CI, 0.58-0.59; P<0.001). In

Table 2 Univariable analysis and Cox proportional hazards regression analysis

\begin{tabular}{|c|c|c|c|c|}
\hline \multirow{2}{*}{ Characteristic } & \multirow{2}{*}{ Univariable analysis $\mathrm{P}$} & \multicolumn{3}{|c|}{ Selected factors for building the model multivariable analysis } \\
\hline & & Hazard ratio & $95 \% \mathrm{Cl}$ & $\mathrm{P}$ \\
\hline Age & $<0.001$ & & & $<0.001$ \\
\hline$<60$ & & 1 (reference) & & \\
\hline $60-70$ & & 1.250 & $1.187-1.316$ & $<0.001$ \\
\hline$\geq 70$ & & 1.676 & $1.586-1.770$ & $<0.001$ \\
\hline Gender & $<0.001$ & & & \\
\hline Male & & 1 (reference) & & \\
\hline Female & & 0.803 & $0.769-0.839$ & $<0.001$ \\
\hline Race & 0.008 & & & \\
\hline White & & - & - & - \\
\hline Black & & - & - & - \\
\hline Other & & - & - & - \\
\hline Location & $<0.001$ & & & 0.016 \\
\hline Upper & & 1 (reference) & & \\
\hline Middle & & 1.025 & $0.925-1.135$ & 0.640 \\
\hline Lower & & 1.104 & $1.055-1.157$ & $<0.001$ \\
\hline Other & & 0.930 & $1.005-0.904$ & 0.930 \\
\hline
\end{tabular}

Table 2 (continued) 
Table 2 (continued)

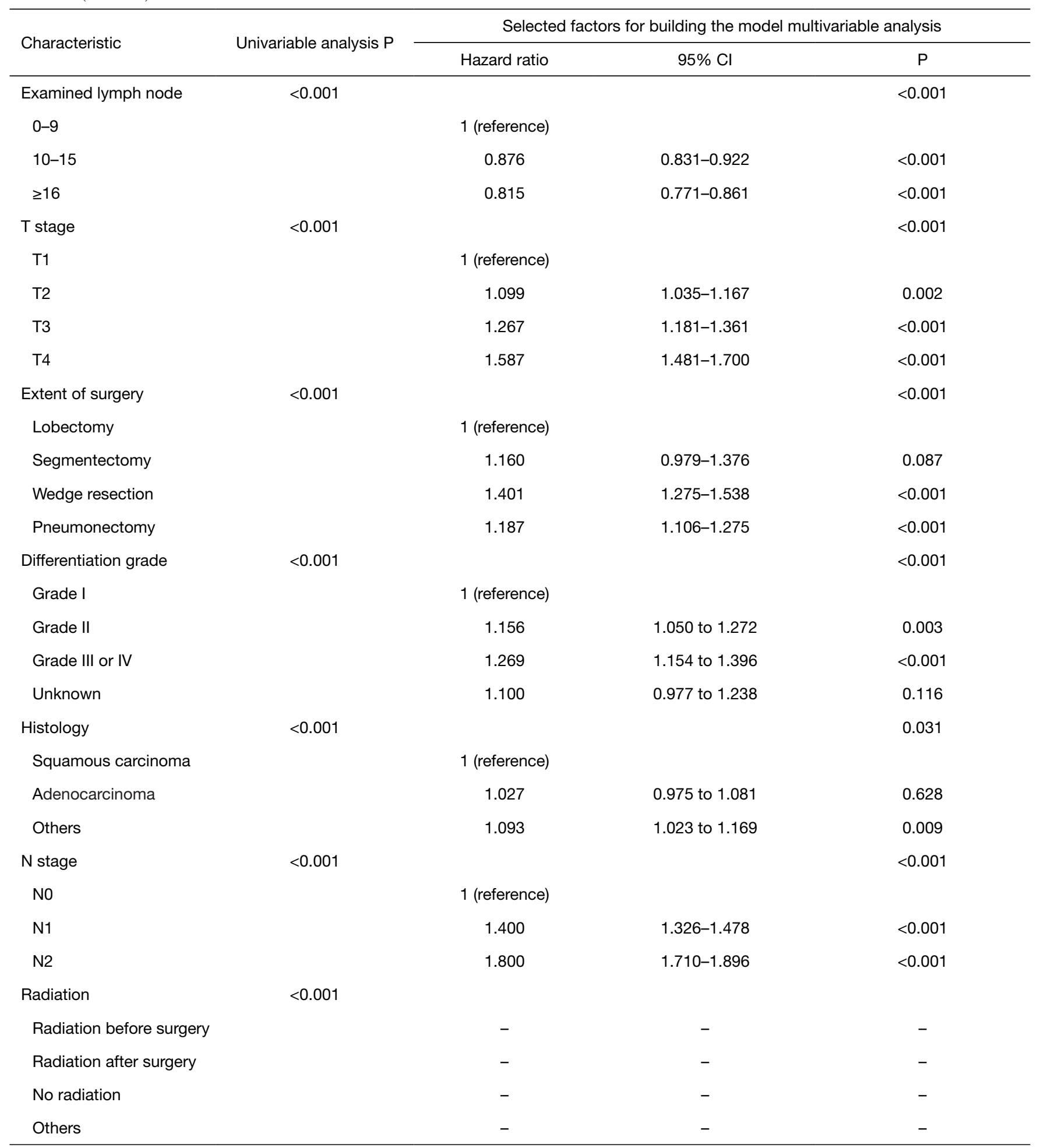




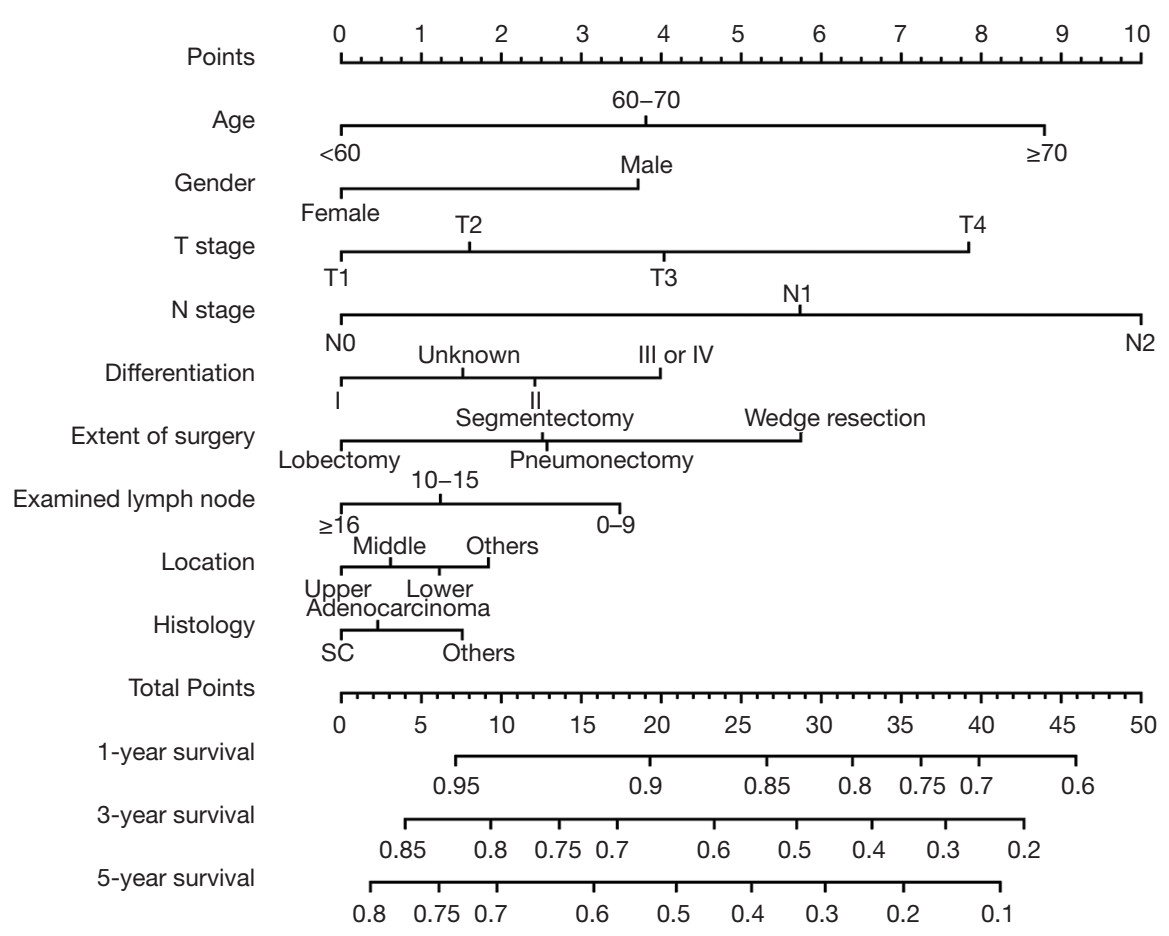

Figure 1 Nomogram predicting 1-, 3-, and 5-year survival for resected NSCLC patients receiving chemotherapy. NSCLC, non-small cell lung cancer.
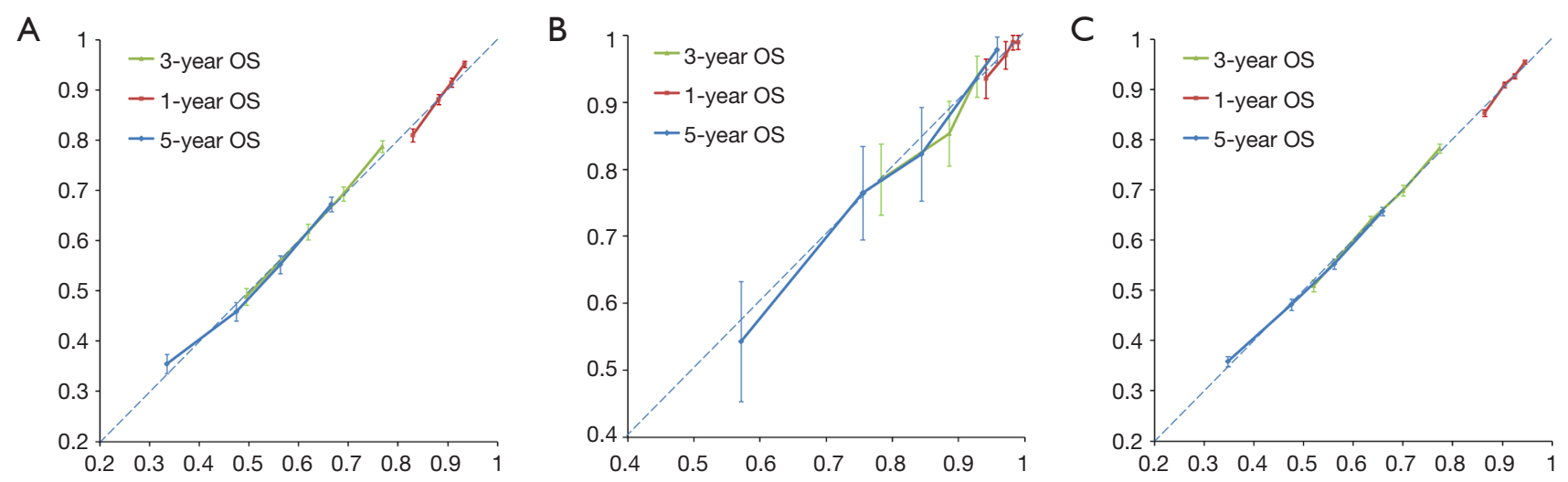

Figure 2 The calibration curves for predicting patient survival at each time point in the training cohort (A), the China external validation cohort (B), and the NCDB external validation cohort (C). NCDB, National Cancer Database.

the China validation cohort, the C-index was also higher for the nomogram prediction $(0.68 ; 95 \% \mathrm{CI}, 0.64-0.71)$ than for the TNM prediction $(0.63 ; 95 \%$ CI, 0.59-0.67), although this difference was not statistically significant $(\mathrm{P}=0.27)$. In the NCDB validation cohort, the $\mathrm{C}$-index was higher for the nomogram prediction $(0.59 ; 95 \% \mathrm{CI}$, $0.58-0.59)$ than for the TNM prediction $(0.57 ; 95 \% \mathrm{CI}$,
$0.566-0.575 ; \mathrm{P}<0.001)$.

\section{Discussion}

As only the $\mathrm{T}$ and $\mathrm{N}$ factors determine the TNM classification for resected NSCLC patients, nomograms with more prognostic factors may be more accurate in 
predicting survival than the TNM classification. Although there are several prognostic models for lung cancer, a nomogram has not been established for patients with resected NSCLC undergoing chemotherapy. Therefore, the aim of this study was to establish a practical survival prediction model for individualized predictions about the survival of these patients. The model was more predictive than the TNM staging system, with superior $\mathrm{C}$-indices.

In this large population study, we discovered that age, sex, primary site, number of dissected lymph nodes, extent of surgery, differentiation, histology, $\mathrm{T}$ stage, and $\mathrm{N}$ stage were independent factors for OS. Most of these factors were consistent with other findings on risk factors for NSCLC $(4,6,13)$. However, no previous study has identified many prognostic factors for patients with resected NSCLC who are receiving chemotherapy $(14,15)$. Despite histology and primary site showing little impact on survival, we tried to incorporate as many clinical prognostic factors into the model as possible to increase its accuracy. Notably, tumors in the upper lobe led to a better prognosis, and tumors in the trachea and translobes are associated with the worst survival. We also found that the extent of surgery and the number of dissected lymph nodes had a considerable impact on survival. This suggests that lobectomy is still the best surgical method to improve prognosis. Additionally, the number of examined lymph nodes is a vital prognostic factor in various kinds of cancer (16-18). Similar studies $(4,6)$ in NSCLC also suggested that a larger number of examined lymph nodes is associated with a better prognosis. Radiation was not included in nomogram as prognostic factor because it is not recommended as standard for resected NSCLC according to the NCCN guidelines. Secondly, we can no obtain radiotherapy data in the external validation cohorts. Therefore, we identified age, gender, $\mathrm{T}$ stage, $\mathrm{N}$ stage, extent of surgery, differentiation grade, histology, location and examined lymph nodes as variables used in the nomogram.

We previously developed a nomogram model with six independent predictors for OS in patients with resected NSCLC (6). Zhang (9) also developed a survival model incorporating four clinical variables to predict OS in earlystage NSCLC patients after surgical resection. In addition, nomograms have also been developed to predict survival after surgery in patients with stage I-II adenosquamous carcinoma (19), in elderly patients with early-stage NSCLC (20), and in patients with IIIA-N2 NSCLC (21) and stage I NSCLC (4). However, chemotherapy factors are not included in these models. Therefore, this study mainly focused on NSCLC patients undergoing chemotherapy. Most patients with resected stage II-III NSCLC receive chemotherapy in the real world in accordance with the NCCN guidelines (2), and some high-risk stage I patients also receive chemotherapy (4). However, a recent study (22) reported that NSCLC patients who received chemotherapy alone achieved a low objective response rate $(29 \%)$; therefore, it is important to predict survival rates to guide other treatment.

In our study, the calibration plots showed excellent agreement between the predicted survival and the actual survival in all cohorts, which confirmed the repeatability and stability of the nomogram. Furthermore, the model also fit the China and NCDB cohorts, which supports the worldwide use of this model, regardless of race or geography. Discrimination was exposed by the considerably superior C-index of the nomogram when compared with the TNM stage in the training cohort and the NCDB external validation cohort. We consider the small sample size to be the key factor to the insignificant difference in $\mathrm{C}$-index in the Chinese cohort $(\mathrm{P}=0.06)$. Moreover, we can also more accurately distinguish and predict the survival of each TNM stage according to the nomogram.

Our study provides one of the first nomograms that can predict OS in patients with resected NSCLC undergoing chemotherapy using a large population-based database. Both doctors and patients will be able to understand individualized survival expectations using the scoring system. This prognostic model provides an instrument for research study and clinical decision, including patient stratification and therapeutic suggestions. In addition, with the development of comprehensive treatment of lung cancer, more treatment methods are needed to improve survival in patients with a low survival rate. A recent study reported (23) that gefitinib plus pemetrexed and carboplatin chemotherapy significantly prolonged survival in EGFRmutant NSCLC. Gandhi et al. (24) found that the addition of pembrolizumab to standard chemotherapy resulted in significantly longer survival than chemotherapy alone in NSCLC patients without EGFR or ALK mutations. Therefore, targeted therapy or immunotherapy may be an option for patients with low survival rates according to the nomogram.

There are still some limitations associated with this study. First, the nomogram was developed using retrospective data from the SEER database, which may introduce the possibility of selection bias. Second, the SEER program lacks data on other prognostic factors, 
such as specific chemotherapy regimens, tumor recurrence, proportion of pathological subtypes, surgical margin status, comorbidity, and molecular factors. In particular, some important molecular factors, including EGFR, ALK, ROS1, c-met, PD-1, and PDL-1, are very important for patient prognosis and treatment. In addition, the SEER data do not distinguish between adjuvant and neoadjuvant chemotherapy. Despite it have some limitations, the nomogram was created applying a large population and was validated using data in two different regions, providing a practical clinical tool to predict survival in patients with resected NSCLC undergoing chemotherapy.

\section{Conclusions}

We developed a prognostic model that can provide individual prediction of OS for patients with resected NSCLC undergoing chemotherapy. Using this model, we can make more precise individualized predictions of the survival of patients after surgery and chemotherapy. This practical nomogram may also support physicians in treatment planning and guide future studies.

The authors are accountable for all aspects of the work in ensuring that questions related to the accuracy or integrity of any part of the work are appropriately investigated and resolved.

\section{Acknowledgments}

Funding: The China State Key Laboratory of Respiratory Disease Independent Subject (Grant No. SKLRDQN-201925); Guangdong Provincial Basic and Applied Basic Research Fund of China (Grant No. 2020A1515110445); Grant 2017YFC0907900/2017YFC0907903 from the National Key R\&D Program of China.

\section{Footnote}

Reporting Checklist: The authors have completed the TRIPOD reporting checklist. Available at http://dx.doi. org/10.21037/tlcr-20-1220

Peer Review File: Available at http://dx.doi.org/10.21037/ tlcr-20-1220

Conflicts of Interest: All authors have completed the ICMJE uniform disclosure form (available at http://dx.doi. org/10.21037/tlcr-20-1220). WL serves as an unpaid associate editor-in-chief from Apr 2018 to Apr 2021. The other authors have no conflicts of interest to declare.

Ethical Statement: The authors are accountable for all aspects of the work in ensuring that questions related to the accuracy or integrity of any part of the work are appropriately investigated and resolved. All procedures performed in this study were in accordance with the Declaration of Helsinki (as revised in 2013).

Open Access Statement: This is an Open Access article distributed in accordance with the Creative Commons Attribution-NonCommercial-NoDerivs 4.0 International License (CC BY-NC-ND 4.0), which permits the noncommercial replication and distribution of the article with the strict proviso that no changes or edits are made and the original work is properly cited (including links to both the formal publication through the relevant DOI and the license). See: https://creativecommons.org/licenses/by-nc-nd/4.0/.

\section{References}

1. Bray F, Ferlay J, Soerjomataram I, et al. Global cancer statistics 2018: GLOBOCAN estimates of incidence and mortality worldwide for 36 cancers in 185 countries. CA Cancer J Clin 2018;68:394-424.

2. National Comprehensive Cancer Network: NCCN Clinical Practice Guidelines in Oncology: NonSmall Cell Lung Cancer. V. 1.2017. Available online: www.ncen.org/ professionals/physician_gls/PDF/nscl.pdf

3. Hamada C, Tsuboi M, Ohta M, et al. Effect of postoperative adjuvant chemotherapy with tegafur-uracil on survival in patients with stage IA non-small cell lung cancer: an exploratory analysis from a meta-analysis of six randomized controlled trials. J Thorac Oncol 2009;4:1511-6.

4. Zeng Y, Mayne N, Yang CJ, et al. A Nomogram for Predicting Cancer-Specific Survival of TNM 8th Edition Stage I Non-small-cell Lung Cancer. Ann Surg Oncol 2019;26:2053-62.

5. Watanabe SI, Nakagawa K, Suzuki K, et al. Neoadjuvant and adjuvant therapy for Stage III non-small cell lung cancer. Jpn J Clin Oncol 2017;47:1112-8.

6. Liang W, Zhang L, Jiang G, et al. Development and Validation of a Nomogram for Predicting Survival in Patients With Resected Non-Small-Cell Lung Cancer. J Clin Oncol 2015;33:861-9.

7. Wang Y, Li J, Xia Y, et al. Prognostic nomogram for 
intrahepatic cholangiocarcinoma after partial hepatectomy. J Clin Oncol 2013;31:1188-95.

8. Zhang Y, Zheng D, Xie J, et al. Development and Validation of Web-Based Nomograms to Precisely Predict Conditional Risk of Site-Specific Recurrence for Patients With Completely Resected Non-small Cell Lung Cancer: A Multiinstitutional Study. Chest 2018;154:501-11.

9. Zhang J, Fan J, Yin R, et al. A nomogram to predict overall survival of patients with early stage non-small cell lung cancer. J Thorac Dis 2019;11:5407-16.

10. Frank E HJR. Regression Modeling Strategies. R Package version 3.4-0. Available online: http://www.rproject.org/

11. Iasonos A, Schrag D, Raj GV, et al. How to build and interpret a nomogram for cancer prognosis. J Clin Oncol 2008;26:1364-70.

12. Hanley JA, McNeil BJ. A method of comparing the areas under receiver operating characteristic curves derived from the same cases. Radiology 1983;148:839-43.

13. Agarwal M, Brahmanday G, Chmielewski GW, et al. Age, tumor size, type of surgery, and gender predict survival in early stage (stage I and II) non-small cell lung cancer after surgical resection. Lung Cancer 2010;68:398-402.

14. Douillard JY, Rosell R, De Lena M, et al. Adjuvant vinorelbine plus cisplatin versus observation in patients with completely resected stage IB-IIIA non-small-cell lung cancer (Adjuvant Navelbine International Trialist Association [ANITA]): a randomised controlled trial. Lancet Oncol 2006;7:719-27.

15. Arriagada R, Dunant A, Pignon JP, et al. Long-term results of the international adjuvant lung cancer trial evaluating adjuvant Cisplatin-based chemotherapy in resected lung cancer. J Clin Oncol 2010;28:35-42.

16. Hua J, Zhang B, Xu J, et al. Determining the optimal number of examined lymph nodes for accurate staging

Cite this article as: Zeng Y, Mayne N, Yang CFJ, Liu J, Cui F, Li J, Liang W, He J; written on behalf of the AME Thoracic Surgery Collaborative Group. A nomogram for predicting overall survival in patients with resected non-small cell lung cancer treated with chemotherapy. Transl Lung Cancer Res 2021;10(4):1690-1699. doi: 10.21037/tlcr-20-1220 of pancreatic cancer: An analysis using the nodal staging score model. Eur J Surg Oncol 2019;45:1069-76.

17. Wang Y, Zhang J, Guo S, et al. Implication of lymph node staging in migration and different treatment strategies for stage T2N0M0 and T1N1M0 resected gastric cancer: a SEER population analysis. Clin Transl Oncol 2019;21:1499-509.

18. Tsai HL, Huang CW, Chen CW, et al. Survival in Resected Stage II Colorectal Cancer Is Dependent on Tumor Depth, Vascular Invasion, Postoperative CEA Level, and The Number of Examined Lymph Nodes. World J Surg 2016;40:1002-9.

19. Li H, Wang Z, Yang F, et al. Development and validation of a nomogram for predicting cancer-specific survival of surgical resected stage I-II adenosquamous carcinoma of the lung. J Surg Oncol 2020;121:1027-35.

20. Sun F, Ma K, Yang X, et al. A nomogram to predict prognosis after surgery in early stage non-small cell lung cancer in elderly patients. Int J Surg 2017;42:11-6.

21. Mao Q, Xia W, Dong G, et al. A nomogram to predict the survival of stage IIIA-N2 non-small cell lung cancer after surgery. J Thorac Cardiovasc Surg 2018;155:1784-92.e3.

22. Langer CJ, Gadgeel SM, Borghaei H, et al. Carboplatin and pemetrexed with or without pembrolizumab for advanced, non-squamous non-small-cell lung cancer: a randomised, phase 2 cohort of the open-label KEYNOTE-021 study. Lancet Oncol 2016;17:1497-508.

23. Noronha V, Patil VM, Joshi A, et al. Gefitinib Versus Gefitinib Plus Pemetrexed and Carboplatin Chemotherapy in EGFR-Mutated Lung Cancer. J Clin Oncol 2020;38:124-36.

24. Gandhi L, Rodríguez-Abreu D, Gadgeel S, et al. Pembrolizumab plus Chemotherapy in Metastatic NonSmall-Cell Lung Cancer. N Engl J Med 2018;378:2078-92. 
Supplementary

Table S1 Histology code and tumor location code

\begin{tabular}{ll}
\hline Location & C34.1-Upper lobe, lung \\
\hline Upper & C34.2-Middle lobe, lung \\
Middle & C34.3-Lower lobe, lung \\
Lower & C34.0-Main bronchus; C34.8-Overlapping lesion of lung; C34.9-Lung, NOS \\
Others & \\
Histology & 8052,8070 to 8073, and 8083 to 8084 \\
Squamous carcinoma & $8140,8230,8254$ to 8255, 8260, 8310, 8333, 8470, 8480, 8481, 8490 8550, 8250, 8252, 8251 and 8253 \\
Adenocarcinoma & \\
Others & \\
\hline
\end{tabular}

